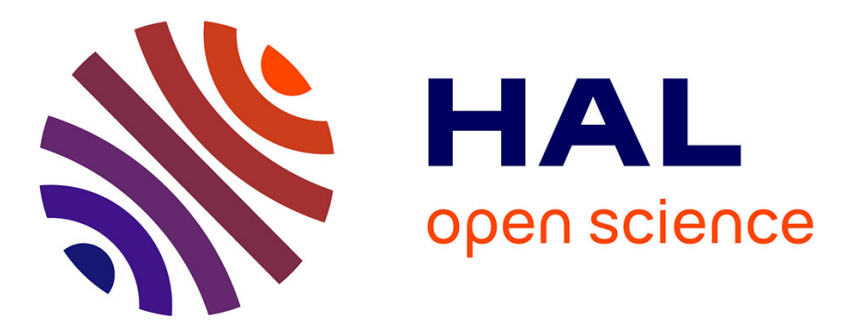

\title{
Laser induced post-desolvation of MALDI clusters
}

Laurent Diologent, Gérard Bolbach, Cristian Focsa, Michael Ziskind, Isabelle Fournier

\section{To cite this version:}

Laurent Diologent, Gérard Bolbach, Cristian Focsa, Michael Ziskind, Isabelle Fournier. Laser induced post-desolvation of MALDI clusters. International Journal of Mass Spectrometry, 2016, 416, pp.29-36. 10.1016/j.ijms.2016.12.005 . hal-01420190

\section{HAL Id: hal-01420190 \\ https://hal.sorbonne-universite.fr/hal-01420190}

Submitted on 20 Dec 2016

HAL is a multi-disciplinary open access archive for the deposit and dissemination of scientific research documents, whether they are published or not. The documents may come from teaching and research institutions in France or abroad, or from public or private research centers.
L'archive ouverte pluridisciplinaire HAL, est destinée au dépôt et à la diffusion de documents scientifiques de niveau recherche, publiés ou non, émanant des établissements d'enseignement et de recherche français ou étrangers, des laboratoires publics ou privés. 


\section{Laser Induced Post-Desolvation of MALDI Clusters}

Laurent Diologent ${ }^{1,2}$, Gérard Bolbach ${ }^{3}$, Cristian Focsa ${ }^{2}$, Michael Ziskind ${ }^{2 *}$, Isabelle Fournier ${ }^{*}$

${ }^{1}$ Univ. Lille, INSERM, U1192 - Laboratoire Protéomique, Réponse Inflammatoire et Spectrométrie de Masse-PRISM, F-59000 Lille, France

${ }^{2}$ Univ. Lille, CNRS, UMR 8523 - PhLAM - Physique des Lasers Atomes et Molécules, F-59000 Lille, France

${ }^{3}$ Sorbonne Universités, UPMC Univ Paris 06, Ecole Normale Supérieure, CNRS, Laboratoire des Biomolecules (LBM), 4 place Jussieu, 75005 Paris, France

Graphical abstract

fx1

\section{Highlights}

- $1064 \mathrm{~nm}$ ns laser for post-desolvation of MALDI clusters is studied

- Laser post-desolvation is shown to increase analyte signal intensity

- Analyte fragmentation is not induced y post-desolvation of MALDI clusters

- Clusters desolvation improves MALDI Sensitivity 


\section{Corresponding Authors:}

Prof. Isabelle Fournier. E-mail: isabelle.fournier@univ-lille1.fr Tel: +33 (0)3 204341 94, Fax: +33 (0)3 20434054 .

Dr. Michael Ziskind. E-mail: michael.ziskind@univ-lille1.fr Tel: +33(0)3 2033 63 30, Fax: +33 (0)3 20336463 


\begin{abstract}
Despite being controversial for many years, it is now widely accepted that the Matrix Assisted Laser Desorption/Ionization (MALDI) process leads to the ejection of aggregates or clusters in addition to isolated particles and ions. Clusters composed of analyte surrounded by matrix molecules exhibit a distribution in size and are relatively stable. Several studies have demonstrated that these clusters do not necessarily fully desolvate before ion extraction, but rather progressively along the ion path. This leads to a decrease in analytical performances since these non-desolvated clusters do not contribute to the ion signal but also generate an important chemical background noise. Therefore, proper cluster desolvation before ion extraction in order to release naked analyte ions in the gas phase holds great promise of improved mass spectra sensitivity. This work presents a set-up allowing cluster desolvation using nanosecond pulses of a second laser that intercepts the expanding MALDI plume before ion extraction.
\end{abstract}

\title{
Key words
}

Matrix Assisted Laser Desorption/Ionization, mechanism, cluster, desolvation 


\section{Introduction}

Matrix-Assisted Laser Desorption Ionization (MALDI) has revolutionized the field of biomolecules analysis thanks to the pioneering work of Hillenkamp and Karas [1,2]. Although very high analytical performances have now been reached, fundamental mechanisms governing the MALDI process are still not fully understood. One illustration is the very different charge distribution shown between MALDI and ElectroSpray Ionization (ESI) [3] despite very similar performances in terms of both sensitivity and molecular weight/polarity of the accessible analytes. It is then clear that a better understanding of these mechanisms is necessary when aiming at optimizing the MALDI source.

Several models have been proposed to explain the features observed in the MALDI spectra. Certain models have focused on the desorption/ablation event [4], others have addressed in more detail the ionization stage [5-7], while some have considered the desorption/ionization event as a continuous integrated process [8-10]. Currently, the model on which instruments are designed is based on the hypothesis that gas phase molecules, including ions produced in MALDI, are individualized and separated from each other. However, because the analyte is diluted in matrix crystals during cocrystallization and is further ejected together with the surrounding matrix molecules, a significant number of analyte-matrix clusters might be present in the plume. Fundamental studies [11-15] and numerical simulations [16-21] have gradually converged towards a comprehensive MALDI model including cluster formation. Numerical simulations have demonstrated the transition from a thermal vaporization regime, characterized by the exclusive ejection of neutrals, to a phase explosion regime with emission of charged compounds and clusters when laser fluence is increased. These results are supported by a series of experiments demonstrating the existence of these clusters using repulsive fields in the source during the delayed extraction [11, 12]. If cluster formation has become a model accepted by the community, its impact on the ionization mechanisms is still under discussion [5, 13]. According to the simulations, clusters are already present in the early desorption phase $(<1 \mathrm{~ns}$ after the laser shot) where they have a broad size distribution. The main problem raised by their presence is that some (possibly many) analyte ions are trapped in these complexes and are being solvated by matrix molecules (by analogy with the mechanisms associated with ESI). Various experiments have shown that the electric field applied to transfer the ions in the mass analyzer (e.g. ion traps IT, Orbitrap, Ion Cyclotron Resonance ICR or Time-of-Flight TOF) contributes to the desolvation of these complexes, which nevertheless remains incomplete. Studies using atmospheric pressure MALDI (AP-MALDI) sources [22] have demonstrated that clusters can be stabilized by AP relaxing collisions. Relaxation is then sufficient to allow for the observation of analyte molecules (M) clustered with matrix molecules (m), resulting in the presence of $[M+n m]^{+}$peaks in the mass spectra. In fact, stabilized clusters were shown to desolvate inside the heated capillary of the instrument under 
AP-MALDI conditions, making possible the observation of multiply charged ions and thus reinforcing the similarity between MALDI and ESI models [23-25]. However, for high-vacuum MALDI, which is still the most sensitive configuration, the experiments have demonstrated that collisions neither effectively stabilize the clusters nor provide their full desolvation. This phenomenon remains a major problem for the quality of the spectra. The naked analyte ions, formed before the ion extraction, will be detected at a time fully defined together by the ejection parameters and the analytical conditions (geometry of the instrument, applied voltages, delayed extraction...). In contrast, there is no direct correlation between the time-of-flight and the $\mathrm{m} / \mathrm{z}$ for clusters which are not fully desolvated before their extraction but continue to desolvate during their acceleration. Clusters with a broad size distribution and long desolvation time appear as unresolved signals and contribute to increase the ("chemical") background noise in MALDI-TOF spectra. This phenomenon has been described by several authors. In particular, the work of Krutchinsky and Chait [26] has pointed out the existence of matrix ions or clusters in the background "noise" when performing MS/MS studies. Specifically, the authors demonstrated using a MALDI-IT instrument the presence of clusters for almost all selected mass-to-charge $(\mathrm{m} / \mathrm{z})$ values and with a lifetime significantly higher than that expected in MALDI-TOF. Sachon et al. [14] studied the desolvation of clusters in MALDI-TOF linear mode by varying the beam deflection voltage. These experiments highlighted, for two different matrices, the existence of a slow component compatible with the hypothesis of desolvation occurring in the source. The same authors showed that the analyte ion was the main fragment released when portions of "chemical background noise" above the analyte $\mathrm{m} / \mathrm{z}$ value were selected for subsequent MS/MS studies. All these studies have demonstrated the limitation of a gradual and partial cluster desolvation during analysis, which results ultimately in a reduced spectral quality due to a concurrent increase in background noise and decrease in analyte signal, especially for high-mass analytes such as proteins. Therefore, achieving full cluster desolvation before their transfer into the analyzer (especially during the delayed extraction occurring before the acceleration for conventional MALDITOF systems) should improve spectral quality. More specifically, spectral sensitivity should be increased because of the rise in analyte signal intensity accompanied by the reduction in background noise.

The aims of this study are twofold: first is to develop and test a system that achieves more efficient cluster desolvation during the MALDI process, and second is to observe the effects of such a postdesolvation on MALDI signals. Taking into account the prominent role of MALDI-TOF systems for analytical purposes, we have developed a system based on a commercial instrument. To achieve effective cluster desolvation on a short time scale (shorter than the extraction delay, i.e. few hundred nanoseconds at most) we use a second pulsed laser beam which intercepts the expanding plume 
perpendicularly in the vicinity of the sample plate surface. The effect of various experimental parameters on the analyte, matrix and cluster signals has been investigated.

\section{Material \&Methods}

\section{Chemicals}

HPLC grade water, ACN and TFA were purchased from BioSolve (Valkenswaard, The Netherlands). Standard calibration peptides Bradykinin ( $\mathrm{Mw}=1060.2$ u.) and ACTH 18-39 (Mw=2465.7 u.), as well as the $\alpha$-cyano 4-hydroxycinnamic acid matrix (HCCA, Mw=189.2 u.) were purchased from Sigma-Aldrich (Saint-Quentin Fallavier, France) and used without further purification.

\section{Sample preparation}

Peptides were dissolved in water to prepare solutions at $5.10^{-6} \mathrm{M}$ for both analytes. The HCCA matrix solution was prepared just before the experiments by dissolving $10 \mathrm{mg} / \mathrm{mL}$ of HCCA in $0.1 \%$ TFA in water:ACN (7:3, v/v). The sample was prepared according to the dried droplet method. Briefly, $1 \mu \mathrm{L}$ of matrix solution and $1 \mu \mathrm{L}$ of analyte were mixed onto the MALDI sample plate. The sample was then left at room temperature and normal pressure conditions until co-crystallization of the matrix/analyte system.

\section{MALDI-TOF MS instrument}

The laser post-desolvation setup was installed on a commercial MALDI-TOF (Voyager Elite system upgraded to STR, PerSeptive Biosystems, Framingham, MA). The instrument is equipped with a delay extraction system and a single stage reflectron, and can be operated both in linear and reflectron modes. The laser used on this instrument is a pulsed $\mathrm{N}_{2}$ laser $(\lambda=337 \mathrm{~nm})$ with a $3 \mathrm{~ns}$ pulse width at a repetition rate of $3 \mathrm{~Hz}$. Two adjacent stages are used for delayed extraction-ion acceleration in the instrument [27]. The $\mathrm{N}_{2}$ laser is focused at $45^{\circ}$ incidence, with an irradiation spot of roughly $200 \mu \mathrm{m}$ size on the sample plate. A neutral density filter placed on the laser beam path outside the chamber insures the control of the delivered laser energy. The maximum output laser energy is $200 \mu \mathrm{J} / \mathrm{pulse}$, this is decreased to 5-15 $\mu \mathrm{J} /$ pulse using the filter for MALDI experiments. Inside the vacuum chamber the sample plate is inserted in a support mounted on an automatic (x,y) plate allowing sample motion under the $\mathrm{N}_{2}$ laser beam. A camera (placed at $45^{\circ}$ with respect to the TOF axis) is used to monitor the sample during experiments.

Experiments are performed by working just above the laser energy threshold allowing analyte ion observation. Conventional MALDI MS spectra of the standard peptides are recorded using both the delayed extraction and reflectron in positive mode. During the delay time $t_{d}$, the first (sample plate) 
and second electrodes are fixed to $23 \mathrm{kV}$. After $\mathrm{t}_{\mathrm{d}}$, the first electrode is switched to a total acceleration of $25 \mathrm{kV}$ and the second electrode is kept to $23 \mathrm{kV}$. $\mathrm{t}_{\mathrm{d}}$ is adjusted in the range $0-200 \mathrm{~ns}$ to provide the highest spectral resolution for the used analyte. It must be noticed that a supplementary electronic delay of 180 ns must be taken into account. Signal at the detector is monitored during the acquisition using a $500 \mathrm{MHz}$ digitizing oscilloscope (2 ns resolution, Tektronix TD520). Recorded spectra are the average of a fixed number of individual spectra (500 laser shots) obtained while moving regularly the sample under the laser beam to achieve good statistics. Recorded spectra are then processed using the Data Explorer v5.0 software.

\section{Setup of the desolvation laser}

A second laser was added on the commercial MALDI-TOF instrument for the purpose of matrix/analyte cluster desolvation. The laser has to be chosen so that it induces the dissociation of matrix clusters and avoids gas phase post-ionization in the meantime [28, 29]. Accordingly, we used a ns pulsed Nd:YAG laser emitting at $1064 \mathrm{~nm}$ (Brillant B, Quantel). Taking into account the ionization potential of the irradiated species (typically 8-9 eV), eight photons are generally required to ionize them, which is highly unlikely in the fluence range used $\left(\sim 320-3200 \mathrm{~J} / \mathrm{m}^{2}\right.$, corresponding to 1-10 $\mathrm{mJ} / \mathrm{pulse}$ ). The second laser was implemented on the side of the instrument allowing the laser beam to enter the source chamber through a newly mounted quartz window. Beam alignment was obtained using two $45^{\circ}$ mirrors and beam collimation to a $2 \mathrm{~mm}$ diameter insured via a telescope setup (Figure 1A). The optical path was set so that the desolvation laser beam enters the source chamber between the sample and the first acceleration grid, since during the delayed extraction time $t_{d}$ the ions are not accelerated (electric field null in the first extraction stage of the source). Ideally, the desolvation laser beam should intercept the MALDI plume in the earlier stage of the desorption/ionization process, just above and parallel to the sample surface and before ions are accelerated. If we consider an average initial axial velocity of $500 \mathrm{~m} / \mathrm{s}$ for HCCA matrix [27, 30-32], the plume material will travel an average of $90 \mu \mathrm{m}$ for $180 \mathrm{~ns}$ delayed extraction time and $500 \mu \mathrm{m}$ if the delay is increased to $1000 \mathrm{~ns}$. Therefore, it is crucial that the laser beam intercepts the plume as close as possible to the sample surface. However, the fact that the second laser entrance window and the sample plate are not in a perfect line of sight, along with the short length of the first acceleration stage $(2.8 \mathrm{~mm})$ require the laser beam to be slightly tilted (Figure 1B). In this configuration, the laser beam hits the edge of the sample plate. A careful alignment of the $1064 \mathrm{~nm}$ laser beam makes sure that the laser hits only the stainless-steel plate and not the MALDI sample. In addition, such irradiation of the stainless-steel plate shows no effect on our studies, since no signal is detected in the mass spectrometer as long as the $337 \mathrm{~nm}$ MALDI laser is turned off. 
Synchronization of the MALDI (337 nm) and desolvation (1064 nm) laser pulses was realized through a 4-channel digital delay pulse generator (Stanford Research System DG 535) controlling the flash lamp $\left(\mathrm{T}_{0}\right)$, the $\mathrm{N}_{2}$ laser irradiation pulse $\left(\mathrm{T}_{0}+\mathrm{t}\right)$, and the Q-Switch of the Nd:YAG laser $\left(\mathrm{T}_{0}+\mathrm{t}+\mathrm{t}^{\prime}\right)$. A frequency divider was added to decrease the $\mathrm{N}_{2}$ laser repetition rate for its synchronization with the Nd:YAG laser, which also decreases the MS instrument duty cycle. The synchronization sequence is presented on Figure 2. During the experiments, 10-100 ns $\mathrm{N}_{2}-\mathrm{Nd}$ :YAG time delays ( $\mathrm{t}$ ') were studied since $t^{\prime}<t_{d}$. In order to provide enough time and space for the MALDI plume to develop while enabling a good interaction between the MALDI plume and the post-desolvation laser, we operated with $\mathrm{t}_{\mathrm{d}}=280 \mathrm{~ns}$.

\section{Results}

The properties (density, composition, dimension, temperature,...) of the MALDI plume evolve spatially and temporally. It is therefore very important to trigger the desolvation laser in such a way that the interaction between the plume and the laser pulse is optimized to intercept the largest possible part of the plume and promote desolvation. We thus first investigated the delay between the laser pulse promoting the MALDI process and the laser set up for the desolvation experiments. Figure 3 displays the evolution of the analyte signal $[\mathrm{M}+\mathrm{H}]^{+}$with the delay between the two laser pulses. A clear increase in the intensity of the protonated ions of both Bradykinin (Figure 3A) and ACTH 1839 (Figure 3B) peptides is evidenced from t'=20 ns up to $100 \mathrm{~ns}$. For both peptides, over a 2-fold increase in signal intensity is observed. This demonstrates a beneficial interaction between the desolvation laser beam and the plume. Assuming an average plume initial velocity of about $500 \mathrm{~m} / \mathrm{s}$ and about $1000 \mathrm{~m} / \mathrm{s}$ for the plume front, and neglecting the time required to establish this velocity, one can roughly estimate that at $t^{\prime}=20 \mathrm{~ns}$ the front plume is localized only $20 \mu \mathrm{m}$ away from the surface of the irradiated sample at the farthest, and the "center" of the plume at about $10 \mu \mathrm{m}$. For $\mathrm{t}^{\prime}=100 \mathrm{~ns}$ the plume front is expected to be located $100 \mu \mathrm{m}$ above the sample surface and the plume "center" at about $50 \mu \mathrm{m}$. Since the plume velocity is only weak at the beginning of the expansion and increases to reach a plateau [33], herein above are the maximum distances that we can expect. These distances are very small compared to the diameter of the desolvation beam $(2 \mathrm{~mm})$. Thus, for $\mathrm{t}^{\prime}=20$ ns, we assume that only the front of the plume interacts with the Nd:YAG laser beam. One can therefore expect that the interaction increases for longer times, which is actually observed in the spectra recorded for a delay between the two lasers of $t^{\prime}=80$ and $100 \mathrm{~ns}$. The evolution of the signals corresponding to the $[\mathrm{m}+\mathrm{H}]^{+}$and $[2 \mathrm{~m}+\mathrm{H}]^{+}$matrix ions contrasts with that of the analytes (Figure 4): the intensity of the matrix peaks varies little over the studied delay range (or in absence of the desolvation beam). More specifically, the $[\mathrm{m}+\mathrm{H}]^{+}$matrix ion shows a slight decrease of intensity until 
a $40 \mathrm{~ns}$ time delay between the two lasers is reached and the $[2 \mathrm{~m}+\mathrm{H}]^{+}$protonated dimer ion is roughly constant over the time range studied. Therefore, the matrix ions show only little effect of the desolvation beam, at least for the selected fluence of $3200 \mathrm{~J} / \mathrm{m}^{2}$. This demonstrates that efficient conditions of interaction between the desolvation laser and the MALDI plume can be obtained.

To deepen our understanding of the observed mechanism, the effect of the desolvation laser fluence $F_{d}$ was studied for different delays between the triggering of the two lasers. Figure 5 shows the evolution of the $[\mathrm{M}+\mathrm{H}]^{+}$ion signal of Bradykinin in the mass spectra for three laser fluences and for a delay of $80 \mathrm{~ns}$ between the two laser pulses. A steady increase in the signal intensity of this ion up to $F_{d} \cong 3200 \mathrm{~J} / \mathrm{m}^{2}$ (highest fluence used in this study) is observed. Importantly, it must be noted that the increase in peak intensity is not accompanied by a deterioration of the spectral resolution. The average intensities of the Bradykinin peaks obtained at different fluences and delays are plotted in Figure 6A. A clear increase in signal intensity is observed above $F_{d} \cong 1000 \mathrm{~J} / \mathrm{m}^{2}$, which strengthens for the highest delay between the two laser i.e. for 80 and $100 \mathrm{~ns}$. Specifically, at $\mathrm{F}_{\mathrm{d}} \cong 3200 \mathrm{~J} / \mathrm{m}^{2}$ and for the shortest delays ( $\mathrm{t}^{\prime}=20$ and $\left.50 \mathrm{~ns}\right)$ the peak recorded is about twice as intense as the peak recorded at zero fluence, i.e. in the absence of the desolvation laser pulse. For the longest delays t'=80 and $100 \mathrm{~ns}$, the peak grows to being about 3 times higher than that recorded at zero fluence. A similar trend is observed for the ACTH 18-39 as shown in Figure 6B. Similarly to Bradykinin, an increase of the ion signal is observed when increasing the fluence of the desolvation laser. Again the intensity of the peak is maximum at the highest desolvation fluence i.e. $F_{d} \cong 3200 \mathrm{~J} / \mathrm{m}^{2}$. At this fluence, a 2fold increase (with respect to the signal recorded without post-desolvation) is observed (depending on the delay t' between the lasers), which is slightly lower than the increase recorded for Bradykinin. This might indicate that desolvation is more difficult for higher molecular weight, ACTH 18-39 molecular weight being more than twice that of Bradykinin. This is well in line with studies demonstrating the increase of cluster size with analyte molecular weight [14]. The evolution of the intensity of the peaks corresponding to the $[\mathrm{m}+\mathrm{H}]^{+}$and $[2 \mathrm{~m}+\mathrm{H}]^{+}$signals of matrix ions is again radically different (Figure 7). For both ions a 2-fold decrease is first observed up to $F_{d}=1500 \mathrm{~J} / \mathrm{m}^{2}$. Above this value, the matrix signal is again increasing. Again the $[\mathrm{m}+\mathrm{H}]^{+}$matrix ion seems more affected by the post-desolvation process than $[2 \mathrm{~m}+\mathrm{H}]^{+}$.

\section{Discussion}

For all experimental conditions investigated in this work a similar desolvation trend is observed. A significant increase in analyte signal intensity is observed above $F_{d}=1000 \mathrm{~J} / \mathrm{m}^{2}$ for all delays between the two lasers. Matrix ions show a less marked dependence on the desolvation laser fluence, slightly decreasing at low fluence and leveling at higher fluences. The clear effect of the desolvation laser on 
the analyte ion signals is in line with a cluster model for MALDI. Indeed, most of the observations can be explained in a model of cluster desolvation during the field free expansion of the MALDI plume. At the maximum desolvation fluence used in this study $\left(3200 \mathrm{~J} / \mathrm{m}^{2}\right)$, a 2 to 3 -fold increase in intensity is observed for the analyte signal whereas the peak width remains strictly unchanged. This suggests the absence of metastable fragmentation in the field-free region. This is in favor of an effective cluster desolvation while preserving the analyte from fragmentation. MALDI clusters are expected to be composed of an analyte surrounded by a large number of matrix molecules. Previous studies $[11,12,14,34]$ suggest that the number of matrix molecules forming the clusters increases with the molecular weight of the analyte. This might explain the lower signal intensity increase observed for ACTH18-39 (Mw=2465.7 u.) compared to Bradykinin ( $\mathrm{Mw}=1060.2 \mathrm{u}$.) even when using a desolvation fluence of $3200 \mathrm{~J} / \mathrm{m}^{2}$. A larger number of matrix molecules would require more laser energy to be efficiently removed from the cluster.

In addition, no significant increase in intensity for matrix ions and no new ion fragments are observed in the spectra. This concurs with no significant analyte fragmentation during plume expansion before ion extraction. In contrast, the slight decrease of the matrix $[\mathrm{m}+\mathrm{H}]^{+}$ion signal at low fluence could be associated with fragmentation processes of a small proportion of the matrix ions. However, at higher fluences, for which an increase in the analyte signal is observed, the matrix signal tends to go back to its initial level. This trend supports a desolvation model releasing free analytes but also matrix ions. In this view, the desolvation could progressively compensate for matrix fragmentation and thus explain the change in behavior observed for the matrix signal, which first decreases before increasing. We could also hypothesize, since we do not observe any features in the spectra supporting a fragmentation process, that desolvation might prevent reneutralization of the analyte ions inside the clusters, which would be in agreement with the lucky survivor model [13-15].

According to the desolvation model, natural cluster desolvation and cluster collisions within the plume with neutrals result in partial to possibly complete cluster desolvation. The change in cluster sizes during ion acceleration, especially for $\mathrm{m} / \mathrm{z}$ above that of the analyte, should yield in the end a signal that is diluted in the background spectra (the so-called chemical noise), and should herein increase the chemical background associated with the analyte signal. Consequently, the use of a postdesolvation laser should reduce the chemical noise. Yet, after careful examination of the spectra, notably the $\mathrm{m} / \mathrm{z}$ region above the analyte signals, we could not evidence any particular change in the background. Therefore, if the desolvation process increases in our experiments, it is likely that this process is far from being complete for all clusters. It is, however, possible that the decrease of the background noise might be observed over a range of $\mathrm{m} / \mathrm{z}$ higher than that recorded [14] or is too weak to be evidenced. Finally, the increase of the analyte signal by fast desolvation after desorption/ionization could also indicate that a higher number of analytes are ionized in the early 
beginning of the desorption and would be reneutralized within the clusters. This would be in line with simulations and experiments showing that laser irradiation results in the ejection of a mixture of individual molecules and molecular aggregates of different sizes [35]. The aggregates require further desolvation to release naked analyte ions. This also indicates that fast desolvation of clusters is favorable to achieve a better sensitivity. This hypothesis is supported by the increased sensitivity reached (at the expense of lower mass resolution) in the continuous extraction mode compared to the delayed extraction, where in the former configuration clusters are immediately accelerated and tend to desolvate through collisions in the dense plume.

\section{Conclusion \& Outlook}

These preliminary studies show that MALDI signals are affected by the use of a post-desolvation IR laser. The results demonstrate a beneficial effect of early cluster desolvation on the analyte signal, which brings about a 2-fold increase in intensity using a fluence of $3200 \mathrm{~J} / \mathrm{m}^{2}$ at $1064 \mathrm{~nm}$. Regarding the matrix, signal evolution with increasing laser fluence suggests that desolvation competes with fragmentation. There would be then a threshold, under which fragmentation dominates and induces the decrease of the matrix peaks in the mass spectrum, and above which cluster desolvation compensates for matrix ion fragmentation. The analyte signals seem not to be affected by the fragmentation processes, probably because of their lower concentration in the plume and the concomitant buffer effect played by matrix molecules. Therefore, we have shown that the release of analyte ions during cluster desolvation significantly improves the sensitivity of the MALDI technique.

This scenario has to be confirmed by increasing the fluence range tested, and by testing the desolvation effect on heavier analytes such as proteins (e.g. Bovine Serum Albumin). Studies involving other laser sources (different wavelengths, pulse durations etc.) could also be carried out in order to amplify the desolvation effect observed or to improve basic understanding of the mechanisms involved. Generally, desolvation requires an energy transfer to the irradiated clusters. This transfer is more effective if the clusters (or the matrix monomer) present an absorption band which coincides with the desolvation laser wavelength. The experiments described here were conducted with a laser which does not satisfy this requirement. Desolvation is then achieved via a purely thermal process whose performance is directly related to the laser fluence employed. A natural extension of this work would be to replicate these experiments with more suitable wavelengths, e.g. in the $2.9-3.4 \mu \mathrm{m}$ spectral range that is in coincidence with the intense $\mathrm{O}-\mathrm{H}, \mathrm{C}-\mathrm{H}$ or $\mathrm{N}-\mathrm{H}$ stretching vibrational bands present in most of the commonly used matrices. This range of wavelength is reachable using a tunable 
IR optical parametric oscillator, such as the one we already used in our group for IR laser resonant desorption of organic molecules embedded in ice matrices [31-35].

\section{Acknowledgements}

Supported by grants from Ministère de l'Enseignement Supérieur et de la Recherche via Institut Universitaire de France (Prof. I. Fournier) and ANR Programme Blanc 2008 ANR-08-BLAN-0026 Fun-MALDI (Dr. G. Bolbach and Prof. I. Fournier).

\section{References}

[1] M. Karas, F. Hillenkamp, Laser desorption ionization of proteins with molecular masses exceeding 10,000 daltons., Anal Chem, 60 (1988) p. 2299-2301.

[2] M. Karas, D. Bachmann, U. Bahr, F. Hillenkamp, Matrix-Assisted Laser Desorption of NonVolatile Compounds, Int. J. Mass Spectrom. Ion Processes, 78 (1987) 53-68.

[3] J.B. Fenn, M. Mann, C.K. Meng, S.F. Wong, C.M. Whitehouse, Electrospray ionization for mass spectrometry of large biomolecules, Science, 246 (1989) 64-71.

[4] K. Dreisewerd, The desorption process in MALDI, Chem Rev, 103 (2003) 395-426.

[5] R. Knochenmuss, Ion formation mechanisms in UV-MALDI, Analyst, 131 (2006) 966-986.

[6] R. Knochenmuss, A quantitative model of ultraviolet matrix-assisted laser desorption/ionization including analyte ion generation, Anal Chem, 75 (2003) 2199-2207.

[7] R. Knochenmuss, R. Zenobi, MALDI ionization: the role of in-plume processes, Chem Rev, 103 (2003) 441-452.

[8] X. Chen, J.A. Carroll, R.C. Beavis, Near-ultraviolet-induced matrix-assisted laser desorption/ionization as a function of wavelength, J. Am. Soc. Mass Spectrom., 9 (1998) 885891.

[9] M. Karas, R. Kruger, Ion formation in MALDI: the cluster ionization mechanism, Chem Rev, 103 (2003) 427-440.

[10] R.C. Beavis, Phenomenological models for matrix $\square$ assisted laser desorption ion yields near the threshold fluence, Organic mass spectrometry, 27 (1992) 864-868.

[11] I. Fournier, A. Brunot, J. Tabet, G. Bolbach, Delayed extraction experiments using a repulsing potential before ion extraction: evidence of non $\square$ covalent clusters as ion precursor in UV 
matrix $\square$ assisted laser desorption/ionization. Part II—Dynamic effects with $\alpha \square$ cyano $\square 4 \square$ hydroxycinnamic acid matrix, Journal of mass spectrometry, 40 (2005) 50-59.

[12] I. Fournier, A. Brunot, J. Tabet, G. Bolbach, Delayed extraction experiments using a repulsive potential before ion extraction: evidence of clusters as ion precursors in UV-MALDI. Part I: dynamical effects with the matrix 2, 5-dihydroxybenzoic acid, Int. J. Mass Spectrom., 213 (2002) 203-215.

[13] M. Karas, M. Gluckmann, J. Schafer, Ionization in matrix-assisted laser desorption/ionization: singly charged molecular ions are the lucky survivors, J Mass Spectrom, 35 (2000) 1-12.

[14] E. Sachon, G. Clodic, T. Blasco, G. Bolbach, Protein desolvation in UV matrix-assisted laser desorption/ionization (MALDI), Journal of the American Society for Mass Spectrometry, 18 (2007) 1880-1890.

[15] T.W. Jaskolla, M. Karas, Compelling evidence for Lucky Survivor and gas phase protonation: the unified MALDI analyte protonation mechanism, J Am Soc Mass Spectrom, 22 (2011) 976988.

[16] L.V. Zhigilei, Y.G. Yingling, T.E. Itina, T.A. Schoolcraft, B.J. Garrison, Molecular dynamics simulations of matrix-assisted laser desorption-connections to experiment, Int. J. Mass Spectrom., 226 (2003) 85-106.

[17] L.V. Zhigilei, P.B. Kodali, B.J. Garrison, Molecular dynamics model for laser ablation and desorption of organic solids, The Journal of Physical Chemistry B, 101 (1997) 2028-2037.

[18] L.V. Zhigilei, B.J. Garrison, Microscopic mechanisms of laser ablation of organic solids in the thermal and stress confinement irradiation regimes, Journal of Applied Physics, 88 (2000) 12811298.

[19] L.V. Zhigilei, Dynamics of the plume formation and parameters of the ejected clusters in shortpulse laser ablation, Applied Physics A, 76 (2003) 339-350.

[20] L.V. Zhigilei, E. Leveugle, B.J. Garrison, Y.G. Yingling, M.I. Zeifman, Computer simulations of laser ablation of molecular substrates, Chemical reviews, 103 (2003) 321-348.

[21] R. Knochenmuss, L.V. Zhigilei, Molecular dynamics model of ultraviolet matrix-assisted laser desorption/ionization including ionization processes, The Journal of Physical Chemistry B, 109 (2005) 22947-22957. 
[22] A. Verentchikov, I. Smirnov, M. Vestal, Collisional cooling and ion formation processes in orthogonal MALDI at intermediate gas pressure, in: Proceedings of the ASMS Conference on Mass Spectrometry and Allied Topics, 1999.

[23] E.D. Inutan, B. Wang, S. Trimpin, Commercial intermediate pressure MALDI ion mobility spectrometry mass spectrometer capable of producing highly charged laserspray ionization ions, Anal Chem, 83 (2011) 678-684.

[24] S. Trimpin, E.D. Inutan, T.N. Herath, C.N. McEwen, Laserspray ionization, a new atmospheric pressure MALDI method for producing highly charged gas-phase ions of peptides and proteins directly from solid solutions, Mol Cell Proteomics, 9 (2010) 362-367.

[25] C.N. McEwen, B.S. Larsen, S. Trimpin, Laserspray ionization on a commercial atmospheric pressure-MALDI mass spectrometer ion source: selecting singly or multiply charged ions, Anal Chem, 82 (2010) 4998-5001.

[26] A.N. Krutchinsky, B.T. Chait, On the nature of the chemical noise in MALDI mass spectra, Journal of the American Society for Mass Spectrometry, 13 (2002) 129-134.

[27] P. Juhasz, M.L. Vestal, S.A. Martin, On the initial velocity of ions generated by matrix-assisted laser desorption ionization and its effect on the calibration of delayed extraction time-of-flight mass spectra, Journal of the American Society for Mass Spectrometry, 8 (1997) 209-217.

[28] A. Rohlfing, A. Leisner, F. Hillenkamp, K. Dreisewerd, Investigation of the desorption process in UV matrix-assisted laser desorption/ionization with a liquid 3-nitrobenzyl alcohol matrix by photoacoustic analysis, fast-flash imaging, and UV-laser postionization $\dagger$, The Journal of Physical Chemistry C, 114 (2009) 5367-5381.

[29] J. Soltwisch, H. Kettling, S. Vens-Cappell, M. Wiegelmann, J. Muthing, K. Dreisewerd, Mass spectrometry imaging with laser-induced postionization, Science, 348 (2015) 211-215.

[30] M. Glückmann, M. Karas, The initial ion velocity and its dependence on matrix, analyte and preparation method in ultraviolet matrix $\square$ assisted laser desorption/ionization, Journal of mass spectrometry, 34 (1999) 467-477.

[31] J. Zhou, W. Ens, K. Standing, A. Verentchikov, B. Sundqvist, Kinetic energy measurements of molecular ions ejected into an electric field by matrix $\square$ assisted laser desorption, Rapid communications in mass spectrometry, 6 (1992) 671-678.

[32] R.C. Beavis, B.T. Chait, Velocity distributions of intact high mass polypeptide molecule ions produced by matrix assisted laser desorption, Chemical physics letters, 181 (1991) 479-484. 
[33] B. Spengler, D. Kirsch, On the formation of initial ion velocities in matrix-assisted laser desorption ionization: Virtual desorption time as an additional parameter describing ion ejection dynamics, Int. J. Mass Spectrom., 226 (2003) 71-83.

[34] S.-H. Lai, K.-H. Chang, J.-L. Lin, C.-L. Wu, C.-H. Chen, Sinapinic acid clusters distribution from monomer to mega Dalton's region in MALDI process, Chemical Physics Letters, 561 (2013) 142-146.

[35] R. Knochenmuss, L.V. Zhigilei, Molecular dynamics simulations of MALDI: laser fluence and pulse width dependence of plume characteristics and consequences for matrix and analyte ionization, J Mass Spectrom, 45 (2010) 333-346. 


\section{Figure Captions}

Figure 1: Schematic representation of the setup realized (top views) for cluster desolvation adding a second pulsed laser (Nd:YAG pulsed, $1064 \mathrm{~nm}$ ). Representation of (A) the laser setup and (B) the laser beam path inside the ion source chamber.

Figure 2: Synchronization time sequence and apparatuses used for the pulsed laser with respect to the MALDI laser and the delay extraction system equipping the instrument.

Figure 3: Evolution of the signal intensity of the $[\mathrm{M}+\mathrm{H}]^{+}$ion of the peptides $(\mathbf{A})$ Bradykinin and (B) ACTH 18-39 with respect to the delay t' between the MALDI laser and the desolvation laser. The fluence of the desolvation laser is set to $3200 \mathrm{~J} / \mathrm{m}^{2}$.

Figure 4: Evolution of the signal intensity of the matrix $(\mathbf{A})[\mathrm{m}+\mathrm{H}]^{+}$and $(\mathbf{B})[2 \mathrm{~m}+\mathrm{H}]^{+}$ion with respect to the delay t' between the MALDI laser and the desolvation laser. The fluence of the desolvation laser is set to $3200 \mathrm{~J} / \mathrm{m}^{2}$.

Figure 5: MALDI mass spectra of Bradykinin recorded for $0,400,1600,3200 \mathrm{~J} / \mathrm{m}^{2}$ desolvation fluences. The delay between the MALDI laser and desolvation laser pulses is set to $80 \mathrm{~ns}$.

Figure 6: Evolution of the signal intensity of the $[\mathrm{M}+\mathrm{H}]^{+}$ion of the peptides (A) Bradykinin and (B) ACTH (18-39) with respect to the desolvation laser fluence for different delays between the MALDI laser and the desolvation laser

Figure 7: Evolution of the signal intensity of the matrix $(\mathbf{A})[\mathrm{m}+\mathrm{H}]^{+}$and $(\mathbf{B})[2 \mathrm{~m}+\mathrm{H}]+$ ion with respect to the desolvation laser energy for different delays between the MALDI laser and the desolvation laser. 

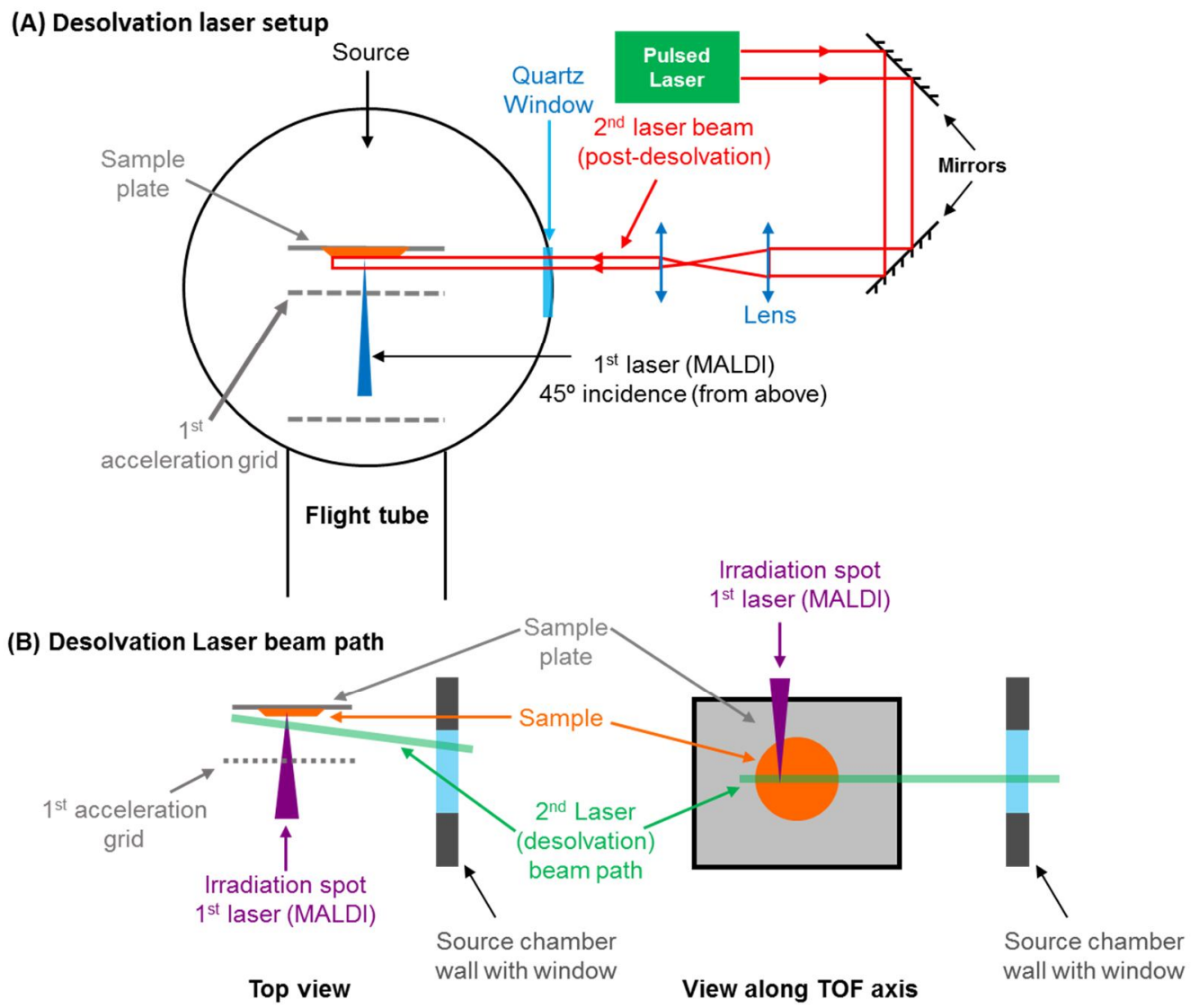

Figure 1 
$\mathrm{Nd}$ :YAG (desolvation)

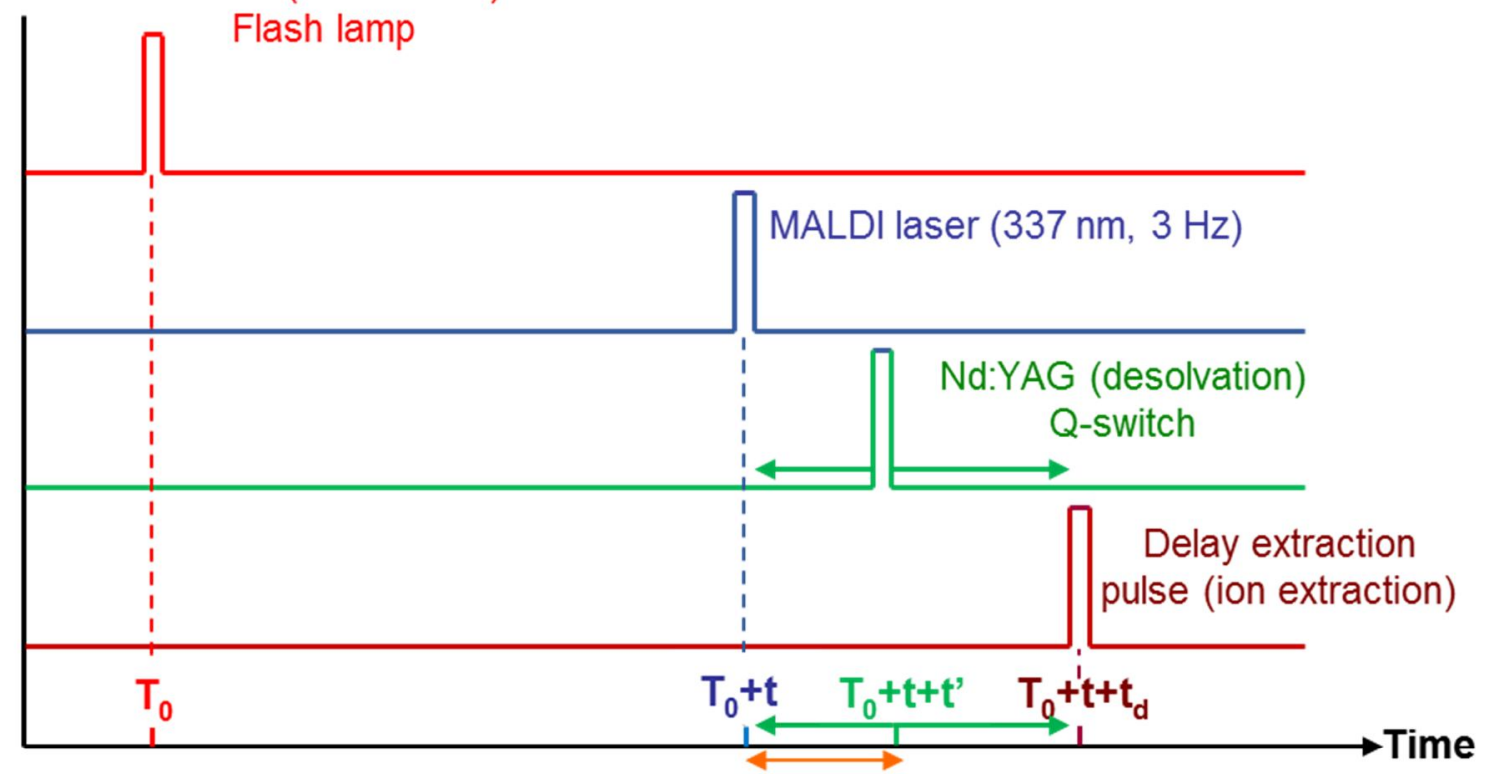

Time-lag between the 2 lasers

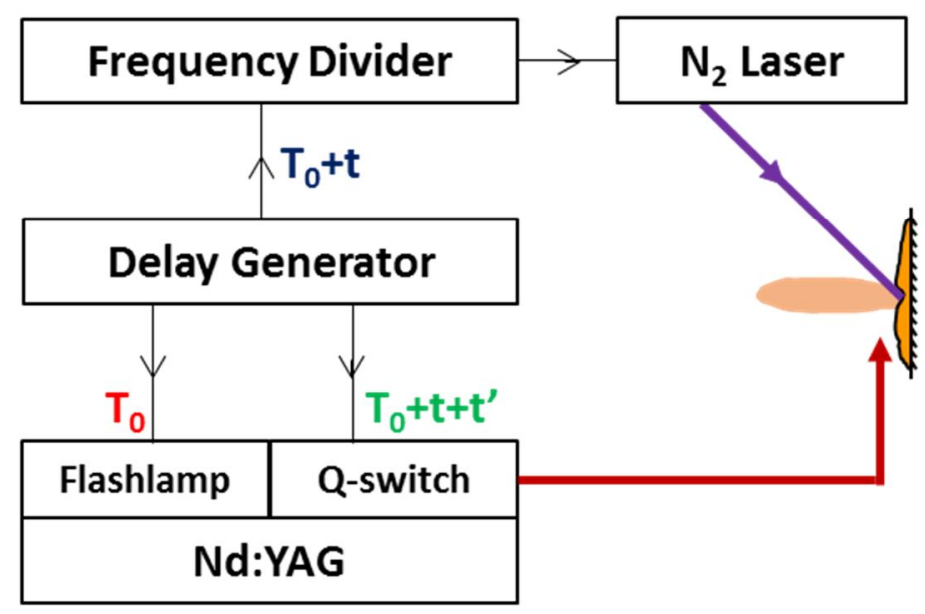

Figure 2 

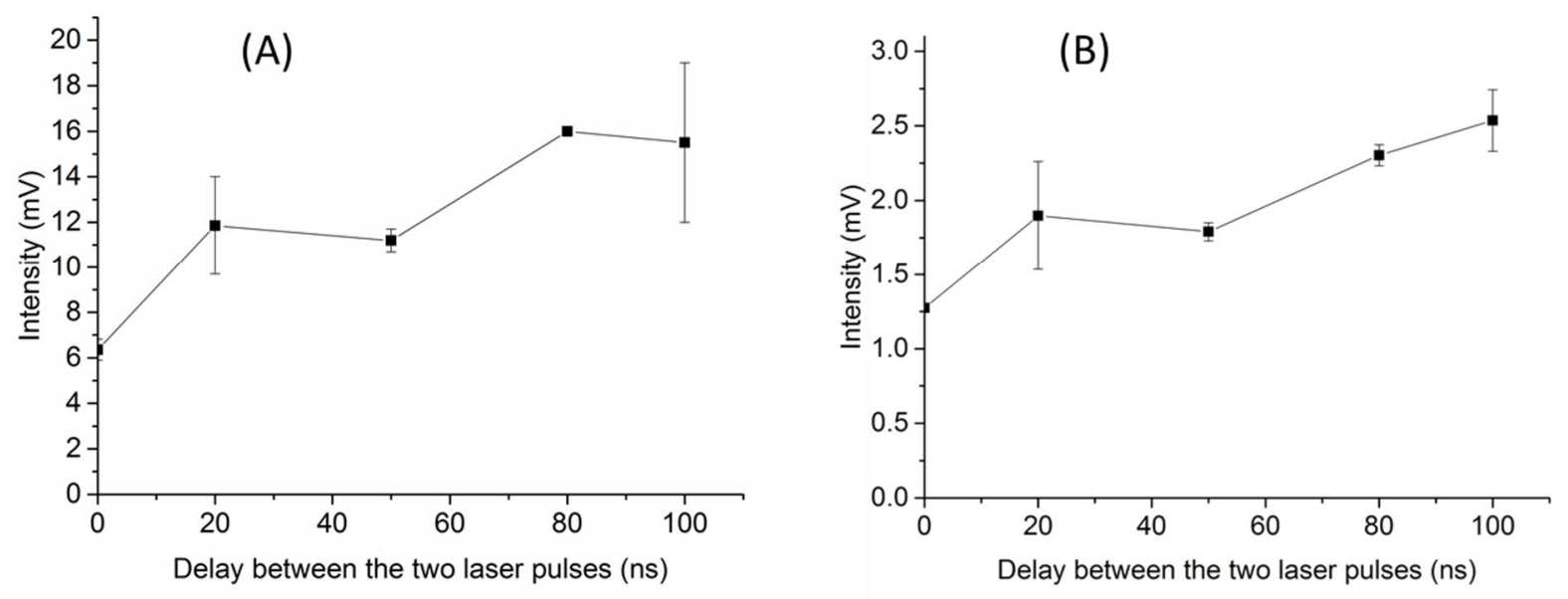

Figure 3 

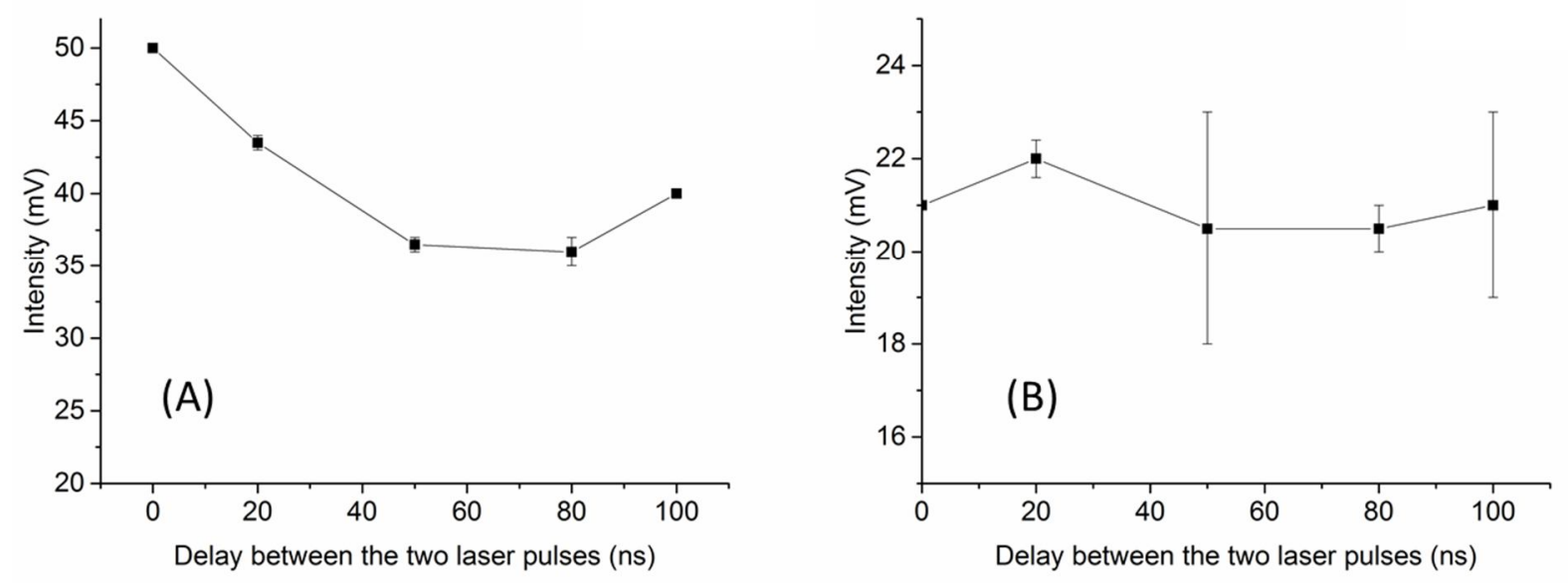

Figure 4 

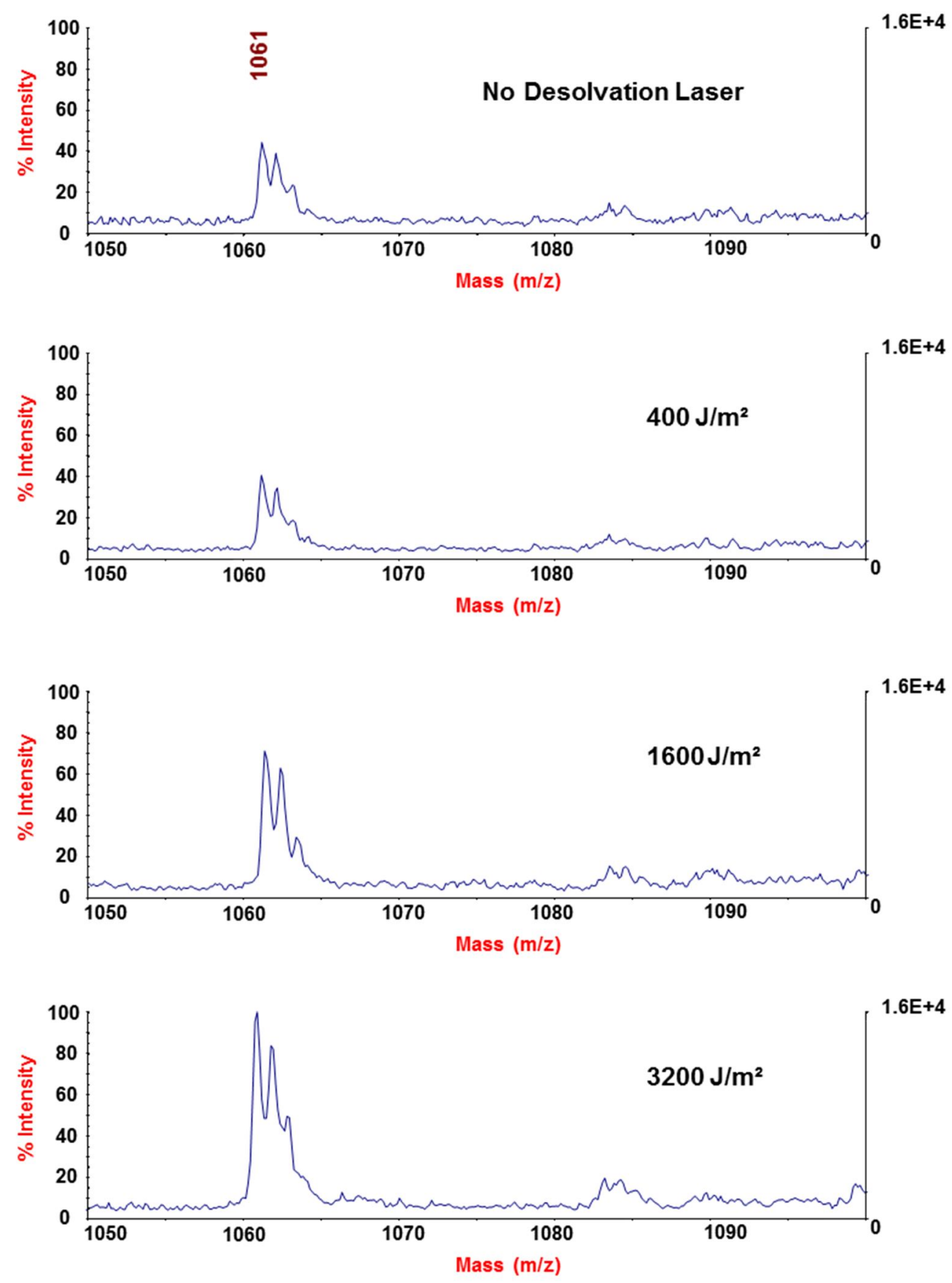

Figure 5 

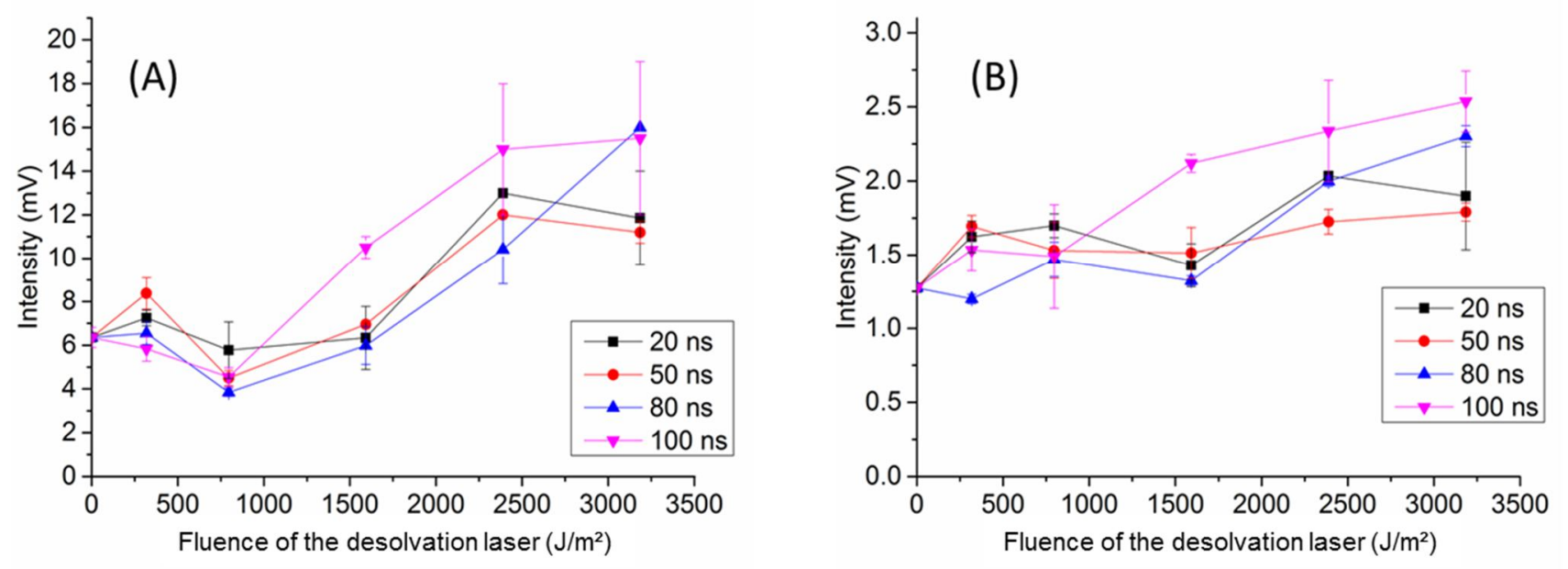

Figure 6 

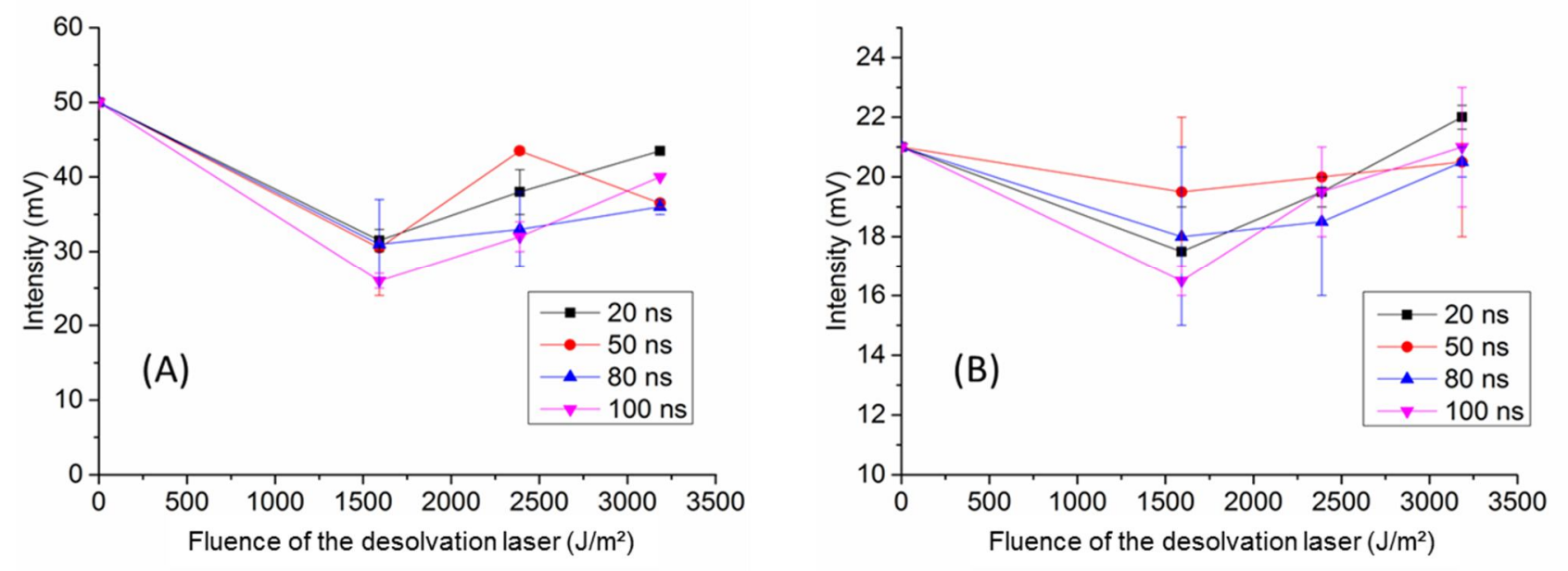

Figure 7 